\title{
Plataforma hardware/software abierta para aplicaciones en procesos de automatización industrial
}

\section{Open Hardware/Software Platform Proposal for Applications in Industrial Automation Processes}

\section{Resumen}

Se presenta una propuesta de implementación de sistemas de control de procesos de bajo costo, que puede ser utilizado por pyme (pequeña y mediana empresa) en Colombia o a nivel educativo para la enseñanza de automatización de procesos industriales. Está compuesta por herramientas abiertas de software y hardware: las plataformas de desarrollo hardware copyleft como parte del proyecto linuxencaja y el sistema de control de procesos open source Proview. Mediante el uso de estos dos proyectos se propone la implementación del esquema cliente/servidor que soporta el protocolo industrial ModBus serial y ModBus sobre Ethernet. El carácter abierto de estos proyectos permite su modificación y uso fácil, la programación de las estrategias de control puede hacerse utilizando un lenguaje de programación gráfica en el que la lógica, secuencias Grafcet y lazos de control pueden ser programados por personal sin experticia.

\section{Palabras clave}

HMI, MODBUS, linuxencaja, SCADA, sistemas de control de proceso, sistemas embebidos.

* Ph. D. Ingeniería Eléctrica, Universidad Nacional de Colombia, Bogotá, Colombia, Magíster en Ingeniería Eléctrica, Universidad de los Andes, Bogotá, Colombia, docente tiempo completo, Universidad Nacional de Colombia, Bogotá, Colombia. Grupo de Microelectrónica de la Universidad Nacional (GMUN). E-mail: cicamargoba@unal.com

** Ms. C. (c). Automatización Industrial, Universidad Nacional de Colombia, Bogotá, Colombia. Ingeniera Electrónica, Universidad Distrital Francisco José de Caldas, Bogotá, Colombia. Grupo de Microelectrónica de la Universidad Nacional (GMUN). E-mail: Iduranb@unal.edu.co

*** Ms.C. Telecomunicaciones, Universidad Nacional de Colombia, Sede Bogotá, Ingeniero Electrónico, Universidad Nacional de Colombia, Bogotá. Director de programa Ingeniería Electrónica e Ingeniería de Telecomunicaciones, Universidad de San Buenaventura, Bogotá. Grupo de Microelectrónica de la Universidad Nacional (GMUN). Email: neferoji@gmail.com 


\section{Abstract}

This work shows a proposal for process control systems implementation at low cost to be acquired by SMEs (Small and Medium Enterprises) in Colombia. It consists of open software and hardware tools: copyleft hardware development platforms from the linuxencaja project and the open source process control system Proview. Joining these two projects allows the implementation of client/server scheme with Modbus support. The open philosophy allows its modification and ease of using; the control strategies could be done using the graphical programming language in which logic, Grafcet sequences and control loops are programmed without expertise.

\section{Keywords}

Embedded systems, HMI, linuxencaja, MODBUS, process control systems, SCADA.

\section{Introducción}

Colombia depende de economías más desarrolladas para el suministro de dispositivos electrónicos para la industria. El sector electrónico del país ha reducido sus actividades de investigación y desarrollo al punto de depender totalmente de productos externos en su mayoría asiáticos; algunos de baja calidad, que no suplen los requerimientos del mercado local, pero utilizados por sus bajos costos [1].

El campo de la automatización en Colombia no es ajeno a este problema. El nivel de desarrollo de soluciones de automatización en el país se limita en gran parte a la venta de equipos y software importados y a la venta de servicios de integración, soporte y mantenimiento. Dichas soluciones se basan en productos propietarios de alto costo [2], a las que solo grandes industrias pueden acceder. Aunque existen soluciones de bajo costo, estas no son integrales y hacen que estos productos no sean competitivos a nivel local.

En Colombia, el 96,4\% de los establecimientos lo constituyen las pymes. Prevalecen las empresas de comercio y servicios, así como las industrias manufactureras y la construcción; con menor participación están la agricultura, ganadería y pesca; las empresas de intermediación financiera y la minería [3]. Estos sectores de la economía son susceptibles a la mejora de sus procesos desde el campo de la automatización.

El diseño de una plataforma hardware/software abierta para aplicaciones industriales de automatización a partir de herramientas abiertas disponibles es el objeto del trabajo de maestría de la autora. Con este sistema de control de bajo costo y altas prestaciones se busca automatizar procesos en pymes para aumentar su productividad y sus niveles de calidad. El artículo presenta los resultados obtenidos hasta el momento en el desarrollo de la misma. 


\section{Antecedentes}

La arquitectura Cliente/Servidor típica de un sistema de control se presenta en la figura 1. Se compone básicamente de una red cliente servidor, una red de control y la instrumentación de campo [7], [8]. La instrumentación de campo comprende sensores digitales como interruptores, fines de carrera, botones de comandos, interruptores de presión, entre otros; sensores análogos como transmisores de presión, flujo, temperatura y actuadores para operar válvulas, bombas, seccionadores, etc.

En la red de control se encuentra el PLC (Controlador Lógico programable) principal, encargado de monitorear y controlar el proceso y adquirir las señales de campo. Este se puede comunicar a través de esta red con otros PLC que pueden a su vez adquirir señales de campo y/o encargarse de subprocesos de la planta. A la red de control se conectan el servidor HMI (Interface Hombre Máquina), el servidor de datos históricos y la estación de ingeniería o desarrollo. La red cliente servidor permite al usuario final controlar y supervisar el proceso y acceder a los datos históricos [9][10].

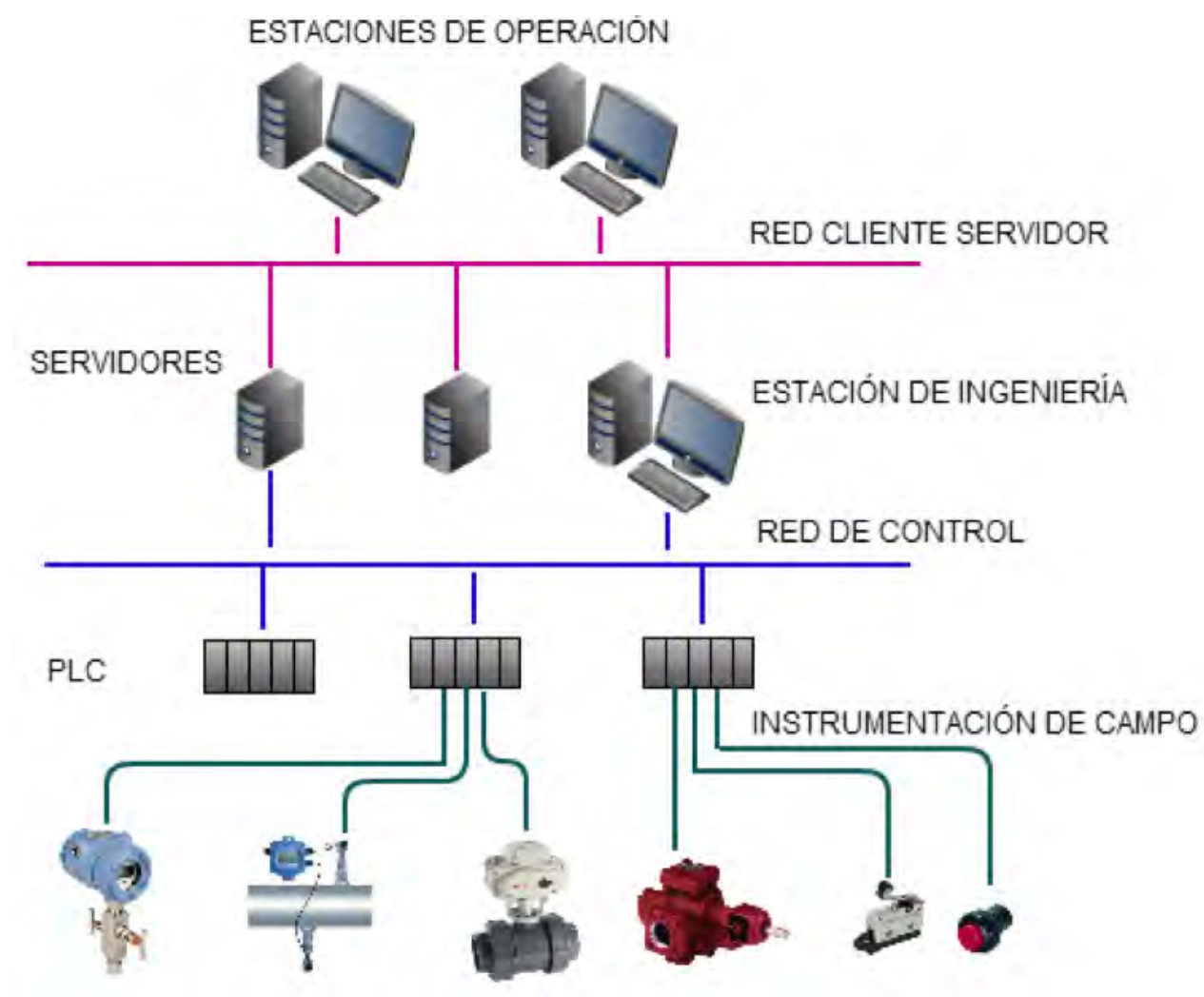

Figura 1. Arquitectura de un sistema de control típico

En el mercado, el precio de un PLC varía desde los 69 USD a los 8900 USD [2] dependiendo de las características del mismo, de las cuales las más importantes son, el número de entradas y salidas disponibles, número de instrucciones que pueden ser programadas, tiempo de scan, puertos de comunicación y protocolos de comunicación. La configuración del PLC de 69 dólares es de 8 entradas digitales, 6 salidas digitales, sin entradas análo- 
gas, programación por puerto serial y con protocolo ModBus RTU para su comunicación con otros equipos [11].

Adicional a este costo se incluye la adquisición del software HMI/SCADA, el cual según la arquitectura requerirá de módulos de software adicionales y según la cantidad de TAG a usar también variará su precio. Un módulo de software HMI/SCADA puede variar entre los 200USD y los 10000 USD. Vale la pena aclarar que al costo de la solución debe agregarse el costo del desarrollo, implementación y mantenimiento del sistema, lo cual hace que para las pymes una solución de este tipo que permite mejorar los índices de desempeño de la producción no sea una opción.

Generar productos bajo el esquema copyleft, eliminaría los problemas de discontinuidad de los productos, permitiría fabricarlos a bajo costo ofreciendo soporte por la comunidad participante en el proyecto. Agilizaría los procesos de adquisición y disponibilidad, pues se fabricaría localmente. Adicionalmente, su producción local estimularía la generación de empleo en nuestro país [21].

El desarrollo de una plataforma física bajo el esquema hardware copyleft compatible con software SCADA libre permitiría a las pequeñas industrias colombianas la automatización de sus procesos a bajo costo y con el respaldo de comunidades a nivel mundial [21], con soluciones reproducibles y modificables según la necesidad específica del usuario. Para ello es necesario definir como mínimo una plataforma hardware a la cual puedan adaptarse interfaces para la comunicación e interfaces para adquisición de señales digitales y análogas; un software para la programación de la plataforma hardware y un software HMI/SCADA.

\section{Proview}

Proview es un sistema de control de procesos Open Source orientado a objetos, basado en Linux y en el concepto de soft-PLC. La arquitectura presentada en la figura 1 puede ser implementada utilizando Proview, donde los PLC son computadoras, de aquí el concepto de soft-PLC. Los módulos de adquisición de señales digitales y análogas se conectan a través de tarjetas $\mathrm{PCl}$. Proview tiene la funcionalidad requerida para realizar control secuencial, ajustes, adquisición de datos, comunicación, supervisión HMI/SCADA, tendencias y almacenamiento de datos [15].

Al utilizar Proview como soft PLC, el tamaño del sistema, sus propiedades y desempeño son limitadas por el sistema operativo anfitrión y su hardware. No existen límites de número de I/O, lazos PID, programas de PLC, contadores, etcétera. El ciclo mínimo de tiempo para un loop de PLC es menor a $1 \mathrm{~ms}$. La comunicación con otros computadores se realiza por Ethernet o por serial.

Proview soporta diferentes protocolos de comunicación como UDP o TCP sockets y Siemens 3964 sobre links seriales. Soporta protocolos de comunicación industrial como de I/O como Profibus/DP, Modbus TCP, OPC [15].

La configuración de un sistema en Proview se realiza gráficamente, por medio de un editor de PLC gráfico y lenguajes de programación de alto nivel como $\mathrm{C}, \mathrm{C}++$, Java o FORTRAN. 
El concepto de Proview está basado en la solución soft-PLC que corre sobre computadores estándar con Linux como sistema operativo [15].

\section{Plataforma Stamps}

Esta plataforma está basada en el procesador i.MX233 de la familia ARM926. Esta tarjeta fue utilizada para el desarrollo de un sistema para medición de calidad de señales eléctricas [16]. La tarjeta está compuesta por un procesador Freescale i.MX233 de $454 \mathrm{MHz}$, un micro controlador STMicroelectronics ARM Cortex M4 con unidad de punto flotante, memoria RAM interna de $256 \mathrm{~KB}$, memoria flash de $1 \mathrm{MB}$, conversores análogo-digital (ADC) de 12 bits y un periférico de comunicación USART. En el procesador STM el cual permite realizar operaciones en tiempo real como soporte de co-procesamiento del i.MX233. EI STM ejecuta el sistema operativo de tiempo real ChibiOS. Sobre el procesador principal es posible implementar un sistema operativo Linux, sobre el cual correrá la aplicación del PLC.

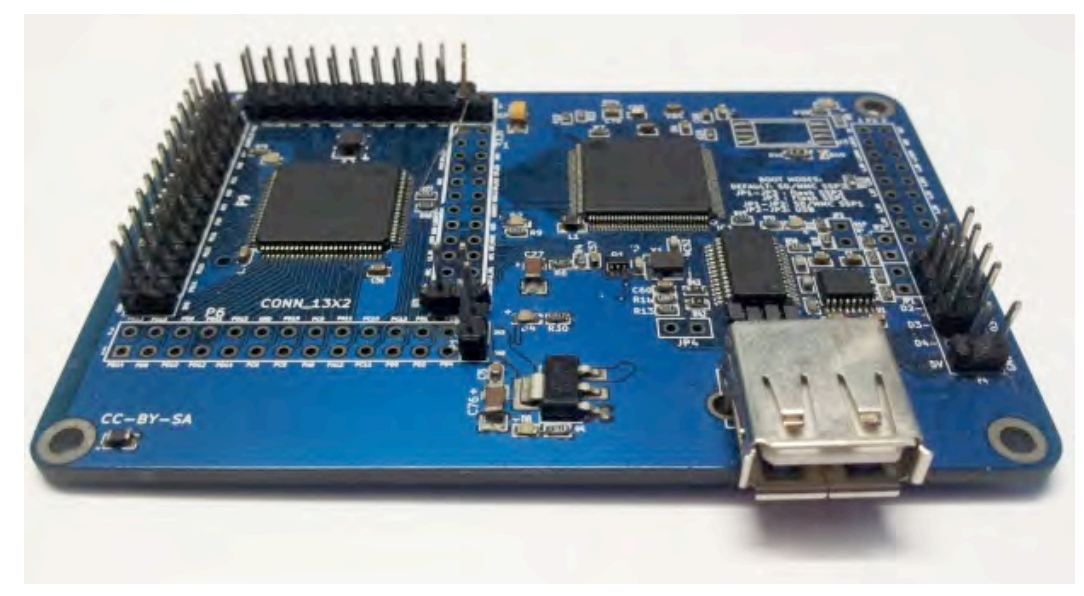

Figura 2. Plataforma de desarrollo Stamps

\section{Plataforma propuesta y prototipo}

La plataforma propuesta es un dispositivo que permite adquirir señales se sensores de una planta ya sean digitales o análogos, monitorear dichas señales y/o procesarlas para ejecutar una acción sobre la planta. La plataforma se programa utilizando lenguaje gráfico Grafcet o bloques de funciones y puede conectarse a una o más estaciones utilizando protocolo ModBus.

Proview en su versión 4.8.0 de mayo de 2012 desarrolló el soporte sobre ARM para compilarlo para sistemas embebidos basados en Linux utilizando compilación cruzada en el ambiente Linux de un sistema operativo anfitrión. Mediante la organización específica de los archivos compilados es posible lanzar Proview sobre ARM y el programa de PLC descrito mediante el ambiente de programación gráfica de Proview [15]. Sobre el procesador ARM i.MX233 de la Stamps, se implementa el kernel de Linux 2.6.35-3 y la distribución de linux buildroot para sistemas embebidos. Proview se compila en el sistema base, se describe el programa de PLC y el servidor cliente ModBus y se configura la compilación para ARM de Proview y el programa de PLC. Los archivos ejecutables de proview y el programa de PLC se copian en el sistema de archivos de la Stamps. 
Para la implementación del cliente y del servidor ModBus sobre la Stamps es necesario tener una interface Ethernet, para lo cual se utiliza el puerto USB de la Stamps conectando un doongle Ethernet, de esta forma es posible asignar una dirección IP a la tarjeta e implementar el cliente o el servidor ModBus.

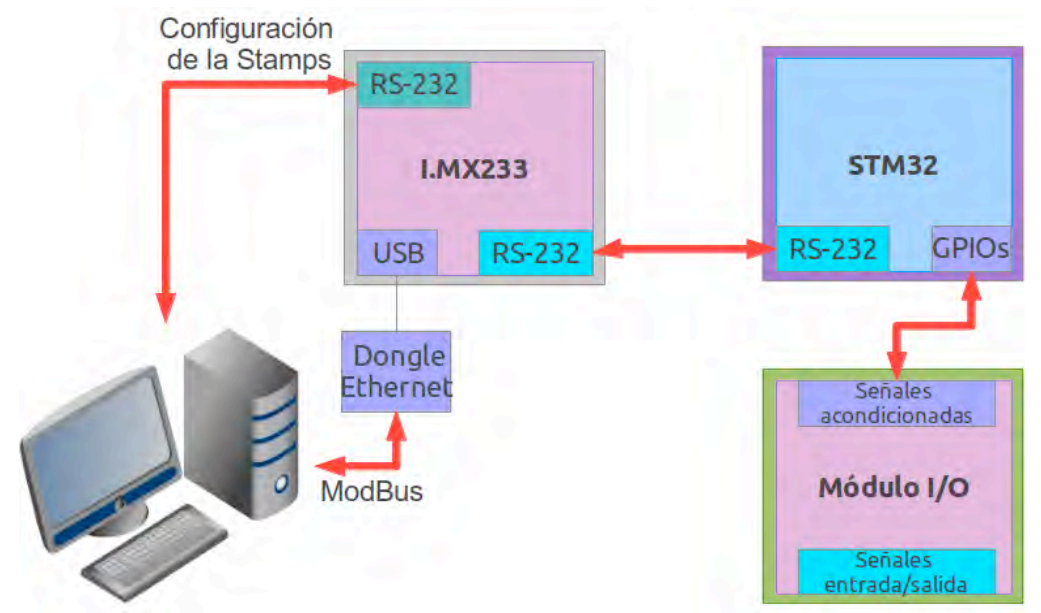

Figura 3. Plataforma hardware/software propuesta

La adquisición de señales se realiza por medio de un módulo hardware diseñado durante el proyecto, alimentado con 12VD y que cuenta con 8 entradas digitales de 0-24VDC de contacto húmedo, 4 entradas análogas 0-5V y 4 salidas digitales 0-24VDC. Este módulo se conecta a los GPIOs del procesador STM32, el cual está operando con ChibiOS, un sistema operativo en tiempo real. Un programa en $\mathrm{C}$ lee los canales análogos del STM32 y los GPIOs configurados como entradas y escribe sobre los GPIOs configurados como salidas. Adicionalmente este programa en C se comunica por puerto RS232 con el procesador i.MX233 y le entrega los datos a la aplicación Proview corriendo sobre el i.MX233.

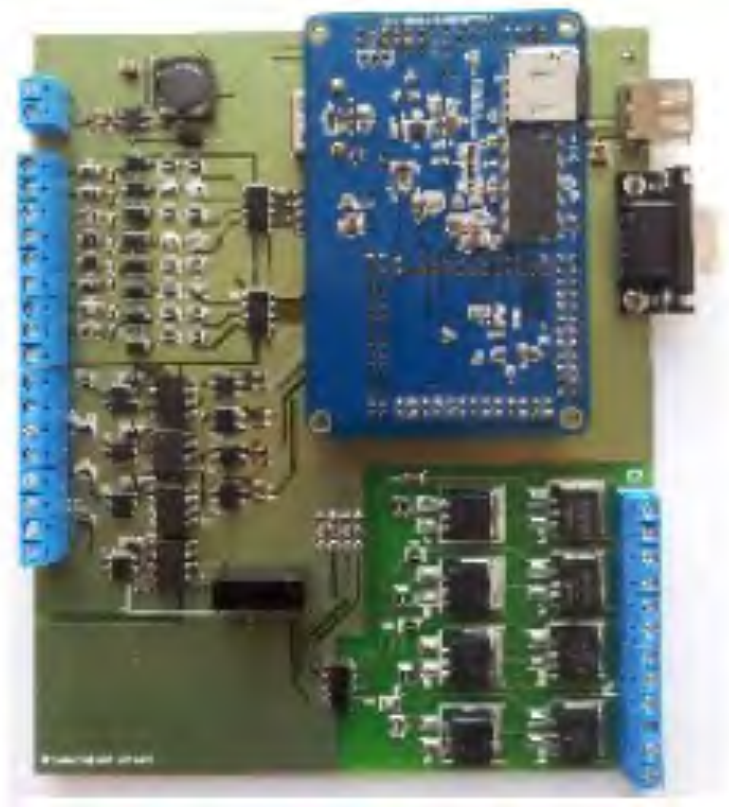

Figura 4. Módulo de entradas/salidas y plataforma Stamps 
De acuerdo con la posibilidad de implementar Proview sobre un sistema embebido basado en ARM se define una arquitectura para sistemas de control de procesos basada en herramientas libres. La arquitectura propuesta se muestra en la figura 5, la cual es una modificación de la arquitectura presentada en la fig. 1 y de la arquitectura de Proview [15]. La arquitectura consta de una red cliente/servidor Ethernet, las estaciones de operación e ingeniería con clientes Proview, la red de control Modbus TCP y las estaciones de proceso. Las estaciones de proceso son las plataformas Stamps corriendo Proview, con módulos de entrada salida para la adquisición de señales de campo.

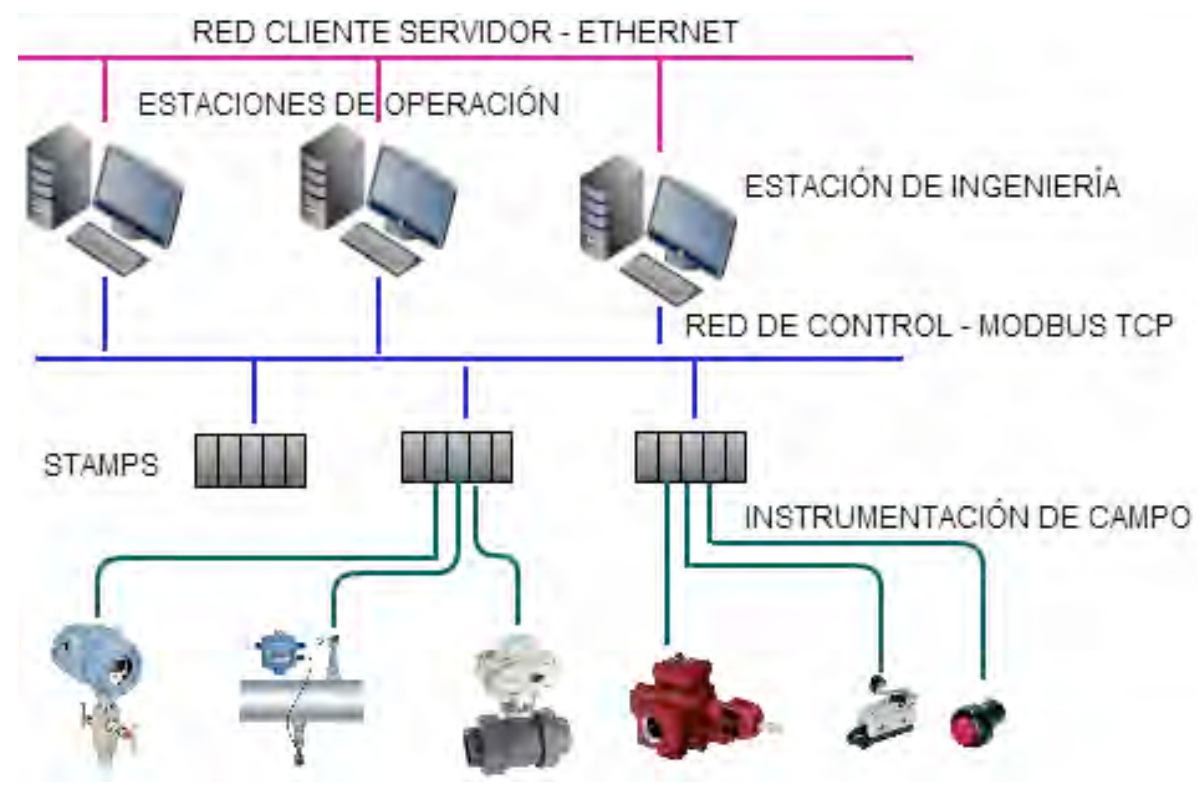

Figura 5. Arquitectura Cliente-Servidor propuesta.

Dentro de los campos de aplicación de la plataforma descrita están el sector de la agroindustria, donde se requieren solución de bajo costo para aplicaciones en sistemas de riego, control climático en invernaderos y agricultura de precisión. En general, un proceso de automatización en la agroindustria tiene cuatro fases que son: la medición de parámetros del cultivo, la interpretación de lecturas, la implementación de algoritmos de control y la modificación de las condiciones del cultivo. El tipo de sensores digitales que se utilizan para este tipo de aplicaciones incluyen caudalímetros, sensores de nivel, bandejas de demanda, sondas de lluvia. Sensores de magnitud análoga como sensores de CE, pH, temperatura, humedad, radiación y dendómetros. Los actuadores modifican o gestionan las condiciones del cultivo, pueden ser motores eléctricos, electroválvulas, calefactores, humidificadores, entre otros.

La domótica es un área de aplicación interesante para la plataforma, donde las necesidades típicas son la seguridad y la comodidad de los habitantes del hogar. El tipo de sensores que se pueden encontrar para seguridad domótica son de detección de intrusos, alarmas de pánico, detección de incendios, detección de fugas de agua los cuales típicamente son sensores digitales, el tipo de controles que se implementan son la desconexión automática de suministros de agua, gas y del servicio eléctrico, control de acceso, control de cortinas y persianas, control de iluminación. 


\section{Implementación}

El primer paso para la implementación de un sistema básico cliente/servidor consiste en instalar Proview 4.8.5-1 sobre un computador anfitrión, el sistema operativo para este caso fue Ubuntu 12.04 English Version. Luego utilizando Proview y un toolchain [17] se compila Proview para ARM de acuerdo con [15]. Seguido de esto se configura el proyecto y se genera el ejecutable. El ambiente de Proview permite configurar el Cliente o el Servidor Modbus gráficamente. Una vez configurado el proyecto de PLC se compila y el ejecutable obtenido (.exe y .load) se copia en el sistema de archivos de la Stamps. Finalmente se lanza Proview sobre la Stamps y se verifica el funcionamiento del servidor Modbus. El procesador STM se programa utilizando Openocd ejecutado sobre el I.MX233, las entradas configuradas como entradas y salidas se conectan al módulo de hardware de entrada y salida.

La Stamps se conecta directamente a la estación de operación implementada en Proview, donde es posible visualizar las señales adquiridas por la plataforma hardware, así como el envío de comandos a las salidas digitales de la plataforma, figura 6.

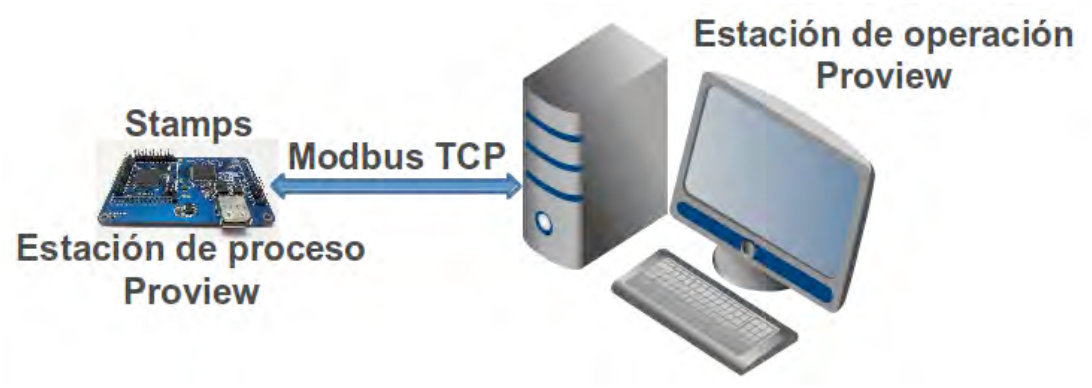

Figura 6. Implementación básica de la plataforma propuesta.

Un ejemplo de implementación básica de la interfaz de Proview se muestra en la figura 7, donde se está monitoreando el estado de dos transmisores de nivel en un sistema de dos tanques en recirculación y se controla el cierre y apertura de dos válvulas así con el encendido y apagado de la bomba en el tanque 2. Los transmisores de nivel son señales analógicas, los controles de las válvulas y la bomba salidas digitales. Es posible visualizar la tendencia de los transmisores así como controlar el encendido y apagado desde los iconos de las válvulas o desde los controles ubicados a la derecha de la pantalla. Gráficamente se observa el nivel de cada tanque por medio de animación y de la escala ubicada al lado de los tanques.

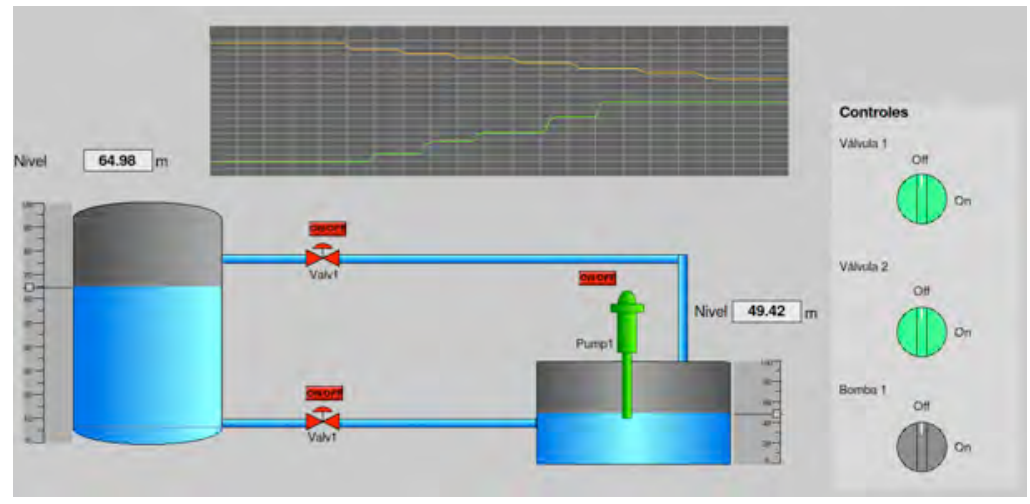

Figura 7. Monitoreo del nivel de dos tanques y control de válvulas 


\section{Conclusiones y trabajo futuro}

Se presentó una aproximación a una plataforma Hardware/Software para control de procesos copyleft, a partir de la cual se propone una arquitectura para el mismo, basada en la tarjeta Stamps del proyecto linuxencaja.

Se implementó un primer módulo de entradas y salidas, para la adquisición de señales y envío de comandos. Como trabajo futuro se propone realizar la implementación de módulos de I/O para aplicación industrial con el fin de adquirir señales y controlar un proceso determinado utilizando la plataforma, de acuerdo con la arquitectura propuesta. Estos módulos deberían incluir además señales análogas 4-20mA, módulos digitales de contacto seco con entradas/salidas configurables por el usuario, entradas digitales con contador rápido, por ejemplo para la conexión de sensores de flujo.

Proview ha implementado el módulo de OPC servidor y cliente, como trabajo futuro se propone explorar la implementación del cliente sobre la Stamps, lo cual resulta muy conveniente para comunicarla con otros $\mathrm{HMI}$ diferentes a Proview que tengan cliente OPC.

Proview tiene una plataforma web para la visualización de datos sobre una estación de proceso (stamps), visualización del programa PLC, visualización de gráficas de proceso, alarmas y eventos, lo cual permite el monitoreo del sistema desde la web.

No fue necesario desarrollar una interfaz de programación para el PLC. Aunque los lenguajes gráficos utilizados en Proview no cumplen con el estándar IEC 61131 -3 son una gran herramienta para la implementación de lógica secuencial aplicada al control de procesos.

A diferencia de las aplicaciones HMI/SCADA open source existentes, esta solución es integral pues reúne hardware y software, permitiendo la implementación completa de un sistema de control de procesos.

\section{Referencias}

[1] C. I. Camargo Bareño, Transferencia tecnológica y de conocimientos en el diseño de sistemas embebidos, Bogotá: Universidad Nacional de Colombia, 2011.

[2] D. Sierk y D. Sierk, «Copyright PLC Compare», 2012. [En línea]. Available: plccompare.com/.

[3] DANE, República de Colombia, «Censo General 2005 Nivel Nacional», Departamento Administrativo Nacional de Estadística, 2005.

[4] Modbus-IDA, «Modbus Application Protocol Specification V1.1b», 2006.

[5] MODICON, Inc., Industrial Automation Systems, "Modbus», North Andover, Massachusetts, 1996.Modbus-IDA, "Modbus Application Protocol Specification V1.1b,» 2006.

[6] Modbus-IDA, «Modbus over Serial Line Specification and Implementation Guide V1.02», 2006.

[7] National Communication System, «Technical Information Bulletin 04-1 Supervisory Control and Data Acquisition (SCADA) Systems». Office of the Manager National Communication System, Arlington, 2004.

[8] K. Stouffer, J. Falco y K. Kent, "Guide to Supervisory Control and Data Acquisition (SCADA) and Industrial Control System Security", National Institute of Technology, Gaithersburg, 2006.

[9] D. Bailey y E. Wright, Practical SCADA for Industry, Perth: Elsevier, 2003.

[10] J. Love, Process Automation Handbook: A guide, Springer, 2007. 
[11] A. Direct, «Automation Direct», 2013. [En línea]. Available: www.automationdirect.com.

[12] «Welcome to Rockwell Automation», 2013. [En línea]. Available: www.rockwellautomation.com.

[13] «The ABB Group - Automation and Power Technologies», 2003. [En línea]. Available: www.abb.com.co.

[14] "Schneider Electric is the Global Specialist in Energy Management», 2013. [En línea]. Available: www.schneider-electric.com.

[15] Sjöfors, R. Karlsson, L. Wirfelt y U. Ljungdahl, «Proview, Open Source Process control», 2013. [En línea]. Available: www. proview.se.

[16] C. I. Camargo Bareño, «linuxencaja», 2012. [En línea]. Available: http://linuxencaja.net.

[17] C. I. Camargo Bareño, «Plataforma Hardware copyleft para la enseñanza de sistemas sigitales», de XVII Workshop de Iberchip, Bogotá, Colombia, 2011.

[18] C. I. Camargo Bareño, «ECBOT y ECB AT91 Plataformas abiertas para el diseño de sistemas embebidos y co-diseño HW/ SW», de VIII Jornada de Computación Reconfigurable y Aplicaciones, Madrid: 2008.

[19] «Qi Hardware Ben Nano Note», 2013. [En línea]. Available: http://en.qi-hardware.com/wiki/Ben_NanoNote.

[20] C. I. Camargo Bareño, «SIE: hardware copyleft como herramienta para la enseñanza de sistemas embebidos», de Congreso Argentino de Sistemas Embebidos CASE 2011, Buenos Aires, 2011.

[21] C. I. Camargo Bareño, «Metodología para la transferencia tecnológica en la industria electrónica basada en software libre y hardware Copyleft», de XVII Workshop de Iberchip, Bogotá, Colombia: 2011.

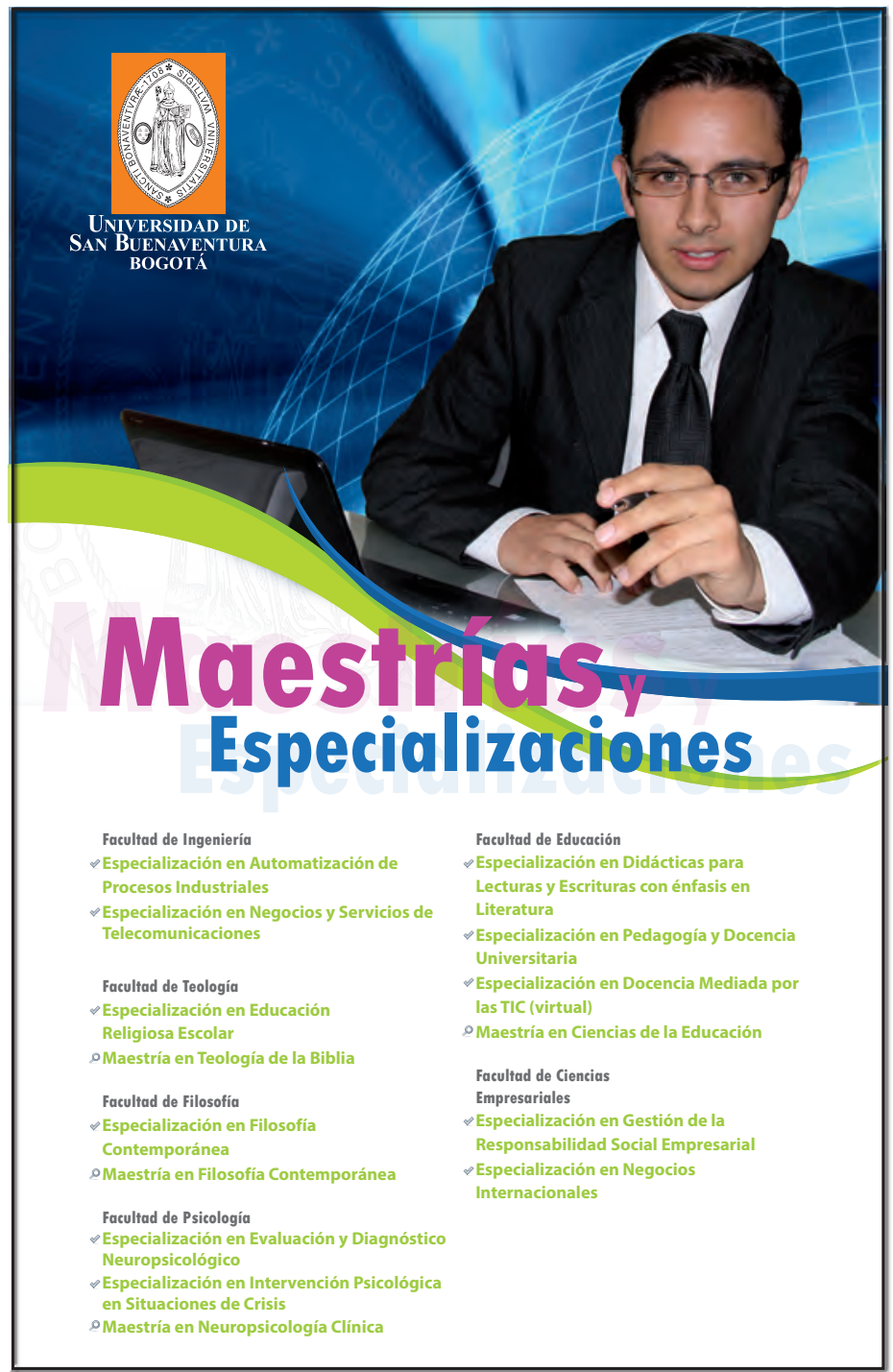

